\title{
Multi-Higgs boson probes of the dark sector
}

\author{
Marvin Flores, ${ }^{1,2}$ Christian Gross $\odot,{ }^{3}$ Jong Soo Kim, ${ }^{4}$ Oleg Lebedev, ${ }^{5}$ and Subhadeep Mondal ${ }^{5}$ \\ ${ }^{1}$ School of Physics, University of the Witwatersrand, 2050 Johannesburg, South Africa \\ ${ }^{2}$ National Institute of Physics, University of the Philippines, Diliman, 1101 Quezon City, Philippines \\ ${ }^{3}$ Dipartimento di Fisica dell'Università di Pisa and INFN, Sezione di Pisa, IT-56127 Pisa, Italy \\ ${ }^{4}$ National Institute for Theoretical Physics and School of Physics, University of the Witwatersrand, 2050 \\ Johannesburg, South Africa \\ ${ }^{5}$ Department of Physics and Helsinki Institute of Physics, Gustaf Hällströmin katu 2a, \\ FI-00014 Helsinki, Finland
}

(Received 5 January 2020; accepted 21 June 2020; published 13 July 2020)

\begin{abstract}
We consider dark sectors with spontaneously broken gauge symmetries, where cascade decays of the dark sector fields naturally produce multi-Higgs boson final states along with dark matter. Our study focuses on two and three Higgs boson final states with missing energy using a multivariate analysis with boosted decision trees. We find that the di-Higgs boson channel is quite promising for the $\bar{b} b+\gamma \gamma$ and $\bar{b} b+\bar{\ell} \ell$ decay modes. The tri-Higgs boson final state with missing energy, on the other hand, appears to be beyond the reach of the LHC in analogous channels. This may change when fully hadronic Higgs boson decays are considered.
\end{abstract}

DOI: $10.1103 /$ PhysRevD.102.015004

\section{INTRODUCTION}

The scalar sector of the Standard Model (SM) remains relatively little explored compared to the gauge sector. In particular, no Higgs boson self-interaction has been measured. It is a well-motivated and experimentally viable possibility that the Higgs field plays the role of a portal into the hidden sector which may contain dark matter and possibly other cosmologically relevant fields [1-3]. Exploring this portal requires Higgs coupling measurements as well as a search for extra states. The Higgs self-coupling can be determined via Higgs boson pair production —one of the central objectives of the future (HL-)LHC searches; cf., e.g., Ref. [4] for a recent summary. Higgs portal dark matter can manifest itself in di-Higgs or multi-Higgs boson final states with large missing energy, which appears due to emission of undetected dark matter. Analogous signals arise in a multitude of new physics models as studied in Refs. [5-20]. LHC searches for di-Higgs $+\mathscr{E}_{T}$ have been presented in Refs. [21-23].

Within a similar framework, a study of di-Higgs $+E_{T}$ production with the $4 b+E_{T}$ final state has been performed in Ref. [24]. It utilizes a jet substructure technique to reconstruct the boosted Higgs bosons. While for heavy dark

Published by the American Physical Society under the terms of the Creative Commons Attribution 4.0 International license. Further distribution of this work must maintain attribution to the author(s) and the published article's title, journal citation, and DOI. Funded by SCOAP. states this method is efficient, in the intermediate mass range (below $700 \mathrm{GeV}$ or so) it is less reliable. In our work, we consider both two and three Higgs boson final states, which subsequently decay into $\bar{b} b, \gamma \gamma$, and $W W$. These have the advantage of being cleaner channels with a lower background. Instead of using a traditional cut-based analysis, we employ (as done for $4 b+E_{T}^{\prime}$ in Ref. [24]) a multivariate technique with boosted decision trees which leads to much better sensitivity to new physics. Further probes of the model are provided by monojet $+E_{T}$ searches [25]. The analysis of Ref. [25] is based on rectangular cuts and as such leads to lower sensitivity than the present study does.

To set the framework for our study, we introduce a simplified model in which cascade decays of heavier states produce dark matter and the Higgs bosons. This model is motivated by non-Abelian gauged hidden sectors in which gauge symmetry is broken completely by vacuum expectation values (VEVs) of a minimal set of dark Higgs boson multiplets [26]. Such models automatically lead to stable dark matter which consists of a subset of gauge fields (and possibly extra scalars). Smaller groups like U(1) and SU(2) $[27,28]$ do not allow for the needed cascade decays, while SU(3) and larger groups have all the necessary features. Further options, e.g., when part of the gauge group condenses, have been explored in Refs. [29,30].

This paper is structured as follows. In Sec. II, we motivate our study and introduce the simplified model. In Sec. III, we perform an LHC study of the di-Higgs boson final state with missing energy using multivariate analysis 
with boosted decision trees and present the resulting sensitivity to model parameters. In Sec. IV, our findings on the tri-Higgs boson final state with missing energy are summarized. Section V concludes our study.

\section{MOTIVATION: MULTI-HIGGS BOSON STATES AND MISSING ENERGY FROM DARK GAUGE SECTORS}

\section{A. Dark Higgsed gauge sectors}

The presence of stable states which can play the role of dark matter (DM) is a common feature of dark sectors with spontaneously broken gauge symmetry. In particular, breaking $\mathrm{SU}(\mathrm{N})$ completely with the minimal number of dark Higgs boson fields automatically leads to stable dark matter. To be specific, let us briefly summarize the main relevant features of the SU(3) example [26] (see also $[31,32])$. The setup contains two dark Higgs boson triplets $\phi_{i}$ to break the symmetry completely. ${ }^{1}$ The Lagrangian is $\mathcal{L}_{\mathrm{SM}}+\mathcal{L}_{\text {portal }}+\mathcal{L}_{\text {hidden }}$, where

$$
\begin{gathered}
-\mathcal{L}_{\mathrm{SM}} \supset V_{\mathrm{SM}}=\frac{\lambda}{2}|H|^{4}+m^{2}|H|^{2}, \\
-\mathcal{L}_{\text {portal }}=|H|^{2}\left(\sum_{i=1}^{2} \lambda_{H i i}\left|\phi_{i}\right|^{2}-\left(\lambda_{H 12} \phi_{1}^{\dagger} \phi_{2}+\text { H.c. }\right)\right), \\
\mathcal{L}_{\text {hidden }}=-\frac{1}{2} \operatorname{tr}\left\{G_{\mu \nu} G^{\mu \nu}\right\}+\sum_{i=1}^{2}\left|D_{\mu} \phi_{i}\right|^{2}-V_{\text {hidden }} .
\end{gathered}
$$

Here, $G_{\mu \nu}=\partial_{\mu} A_{\nu}-\partial_{\nu} A_{\mu}+i \tilde{g}\left[A_{\mu}, A_{\nu}\right]$ is the field strength tensor of the $\mathrm{SU}(3)$ gauge fields $A_{\mu}^{a}$ with gauge coupling $\tilde{g}$, $D_{\mu} \phi_{i}=\partial_{\mu} \phi_{i}+i \tilde{g} A_{\mu} \phi_{i}$, and $H$ is the Higgs doublet, which in the unitary gauge can be written as $H^{T}=(0, v+h) / \sqrt{2}$. The potential for the dark Higgs bosons is given by

$$
\begin{aligned}
V_{\text {hidden }}\left(\phi_{1}, \phi_{2}\right)= & m_{11}^{2}\left|\phi_{1}\right|^{2}+m_{22}^{2}\left|\phi_{2}\right|^{2}-\left(m_{12}^{2} \phi_{1}^{\dagger} \phi_{2}+\text { H.c. }\right) \\
& +\frac{\lambda_{1}}{2}\left|\phi_{1}\right|^{4}+\frac{\lambda_{2}}{2}\left|\phi_{2}\right|^{4}+\lambda_{3}\left|\phi_{1}\right|^{2}\left|\phi_{2}\right|^{2} \\
& +\lambda_{4}\left|\phi_{1}^{\dagger} \phi_{2}\right|^{2} \\
& +\left[\frac{\lambda_{5}}{2}\left(\phi_{1}^{\dagger} \phi_{2}\right)^{2}+\lambda_{6}\left|\phi_{1}\right|^{2}\left(\phi_{1}^{\dagger} \phi_{2}\right)\right. \\
& \left.+\lambda_{7}\left|\phi_{2}\right|^{2}\left(\phi_{1}^{\dagger} \phi_{2}\right)+\text { H.c. }\right] .
\end{aligned}
$$

In the unitary gauge, $\phi_{1}$ and $\phi_{2}$ can be written as

\footnotetext{
${ }^{1}$ This is the minimal scalar field content needed to break SU(3) completely. With fewer scalars, part of the gauge group would remain unbroken and may eventually condense, leading to different phenomenology; cf. [29,30].
}

TABLE I. $Z_{2} \times Z_{2}^{\prime}$ parities of the scalars and dark gauge bosons.

\begin{tabular}{lc}
\hline \hline Fields & $Z_{2} \times Z_{2}^{\prime}$ \\
\hline$h, \varphi^{1}, \varphi^{2}, \varphi^{3}, A_{\mu}^{7}$ & $(+,+)$ \\
$A_{\mu}^{2}, A_{\mu}^{5}$ & $(-,+)$ \\
$A_{\mu}^{1}, A_{\mu}^{4}$ & $(-,-)$ \\
$\varphi^{4}, A_{\mu}^{3}, A_{\mu}^{6}, A_{\mu}^{8}$ & $(+,-)$ \\
\hline \hline
\end{tabular}

$$
\begin{aligned}
& \phi_{1}=\frac{1}{\sqrt{2}}\left(\begin{array}{c}
0 \\
0 \\
v_{1}+\varphi_{1}
\end{array}\right), \\
& \phi_{2}=\frac{1}{\sqrt{2}}\left(\begin{array}{c}
0 \\
v_{2}+\varphi_{2} \\
v_{3}+\varphi_{3}+i\left(v_{4}+\varphi_{4}\right)
\end{array}\right),
\end{aligned}
$$

respectively, where the $v_{i}$ are real VEVs and $\varphi_{i}$ are real scalar fields.

For simplicity, we assume an unbroken $C P$ symmetry in the scalar sector; i.e., we assume that the couplings are real and $v_{4}=0 .^{2}$ As explained in Refs. [26,34], the model then has an unbroken $\mathrm{U}(1) \times Z_{2}^{\prime}$ global symmetry. Its $Z_{2} \times Z_{2}^{\prime}$ subgroup leads to stability of multicomponent DM. The parities of the fields under $Z_{2} \times Z_{2}^{\prime}$ transformations are summarized in Table I.

The lightest states with nontrivial parities cannot decay to the Standard Model (SM) particles and are viable DM candidates. The hidden vector and scalar fields can mix with other fields of the same $Z_{2} \times Z_{2}^{\prime}$ parity.

In this example, $A_{\mu}^{1-3}$ and $\varphi^{4}$ play the role of dark matter. The other vectors $A_{\mu}^{4-8}$ are heavier and decay into $A_{\mu}^{1-3}$ with scalar emission. The scalar couplings to the vectors depend on the VEVs of the triplets as well as their mixing with the SM Higgs boson. The $C P$-even scalars are all expected to mix, and their mass eigenstates include the $125 \mathrm{GeV}$ Higgs boson $h$, a heavier scalar $H$, and two more heavy scalars which play no significant role in our discussion. Given that $H$ emission is often kinematically forbidden, cascade decays of the heavy states naturally produce multi-Higgs boson $h$ signals.

\section{B. Simplified model}

In our study, only main features of this setup play a role. Hence, it is convenient to introduce a simplified model which inherits salient features of the Higgs portal framework. Consider an extension of the SM by three fields: a Higgs-like scalar $H$ with mass $m_{H}$, a stable vector field $A_{L}$ with mass $m_{A_{L}}$, and a heavier unstable vector field $A_{H}$ with mass $m_{A_{H}}$. Dark matter is assumed to be composed of $A_{L}$,

\footnotetext{
${ }^{2}$ If this assumption is relaxed, the model still has dark matter which consists of a subset of stable states considered here [33].
} 

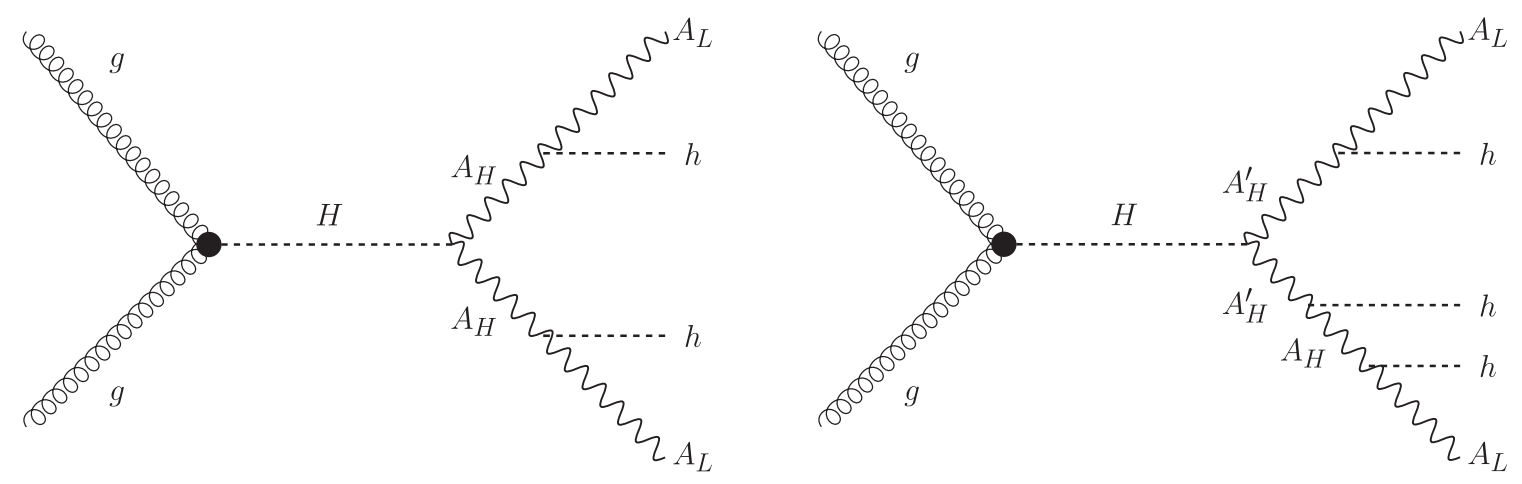

FIG. 1. Left: $h h+E_{T}$ production. Right: $h h h+E_{T}$ production.

while the $125 \mathrm{GeV}$ Higgs boson $h$ and the heavier Higgs boson $H$ are mixtures of the SM Higgs doublet and the hidden sector singlet characterized by the mixing angle $\theta$.

The interactions of the prototype fields are listed in Ref. [26]. The result depends on which of the triplets the SM Higgs boson mixes with predominantly. For our purposes, it is convenient to parametrize the couplings in terms of the mixing angles as follows. The couplings of $h$ and $H$ to SM matter and SM gauge fields are given by

$$
\begin{aligned}
\mathcal{L} \supset & (h \cos \theta+H \sin \theta) \\
& \times \frac{1}{v}\left[2 m_{W}^{2} W_{\mu}^{+} W^{\mu-}+m_{Z}^{2} Z_{\mu} Z^{\mu}-\sum_{f} m_{f} \bar{f} f\right],
\end{aligned}
$$

while the couplings of $h$ and $H$ to $A_{L}$ and $A_{H}$ are given by

$$
\begin{aligned}
\mathcal{L} \supset & (-h \sin \theta+H \cos \theta) \\
& \times \frac{\tilde{g}}{2}\left[\cos \delta m_{A_{L}}\left(A_{L}^{\mu}\right)^{2}+\sin \delta m_{A_{H}}\left(A_{H}^{\mu}\right)^{2}\right] .
\end{aligned}
$$

In addition to the masses, the new physics parameters are the dark gauge coupling $\tilde{g}$, the Higgs mixing angle $\theta$, and an angle $\delta$ that sets the relative strength of the scalar coupling to the dark vectors. ${ }^{3}$ Further relevant couplings include the terms

$$
\mathcal{L} \supset-\kappa_{112} \frac{v}{2} H h^{2}-\sigma\left(A_{L}\right)_{\mu} A_{H}^{\mu} h .
$$

Here $\kappa_{112}$ is not fixed by the other model parameters, so that we can take $\operatorname{BR}(H \rightarrow h h)$ to be a free variable. The $\sigma$ term, which is also a free parameter within the simplified model, accounts for decay of the heavy gauge boson into dark matter and SM states.

The di-Higgs $+E_{T}$ final state is generated through the diagram in Fig. 1, left. We consider the regime where $H$ is produced on shell and decays into a pair of $A_{H}$ with a

\footnotetext{
${ }^{3}$ Within the UV complete model of the previous subsection, $\sin \delta$ depends on the composition of the heavy Higgs boson $H$ in terms of the $C P$-even dark scalars.
}

significant branching fraction. These subsequently decay into $A_{L}$ 's and $h$ 's as long as kinematically allowed, while the $A_{L}$ pair escapes undetected, thereby producing the missing energy signal.

In the simplest model, $\operatorname{BR}\left(A_{H} \rightarrow A_{L}+h\right) \simeq 100 \%$ for $m_{A_{H}}>m_{A_{L}}+m_{h}$. On the other hand, $\operatorname{BR}\left(H \rightarrow A_{H} A_{H}\right)$ varies. The partial width for the $H$ decay into SM fermions and SM vector bosons is given by

$$
\Gamma\left(H \rightarrow f \bar{f}, Z Z, W^{+} W^{-}\right)=\sin ^{2} \theta \times\left.\Gamma_{\mathrm{SM}}\right|_{m_{h} \rightarrow m_{H}} .
$$

The partial widths for the $H$ decay to hidden vectors are given by

$$
\begin{gathered}
\Gamma\left(H \rightarrow A_{L} A_{L}\right)=\frac{\tilde{g}^{2} \cos ^{2} \theta \cos ^{2} \delta m_{H}^{3}}{128 \pi m_{A_{L}}^{2}} f\left(x_{L}\right), \\
\Gamma\left(H \rightarrow A_{H} A_{H}\right)=\frac{\tilde{g}^{2} \cos ^{2} \theta \sin ^{2} \delta m_{H}^{3}}{128 \pi m_{A_{H}}^{2}} f\left(x_{H}\right),
\end{gathered}
$$

where $x_{L / H} \equiv 4 m_{A_{L / H}}^{2} / m_{H}^{2}$ and $f(x)=\left(1-x+3 x^{2} / 4\right) \sqrt{1-x}$. Figure 2 shows the variation of $\operatorname{BR}\left(H \rightarrow A_{H} A_{H}\right)$ with $m_{A_{H}}$ and $\cos \delta$. The other parameters are set to $\sin \theta=0.3, \tilde{g}=2$, $m_{H}=600 \mathrm{GeV}$, and $m_{A_{L}}=10 \mathrm{GeV}$, and $\mathrm{BR}(H \rightarrow h h)$ is kept fixed at 0.2. This choice is motivated by having a sizable production cross section for $H$ : Both $\sin \theta$ and $\tilde{g}$ should be substantial, while $H$ should not be too heavy. We see that $\cos \delta$ is required to be small in order to get a significant $\operatorname{BR}\left(H \rightarrow A_{H} A_{H}\right)$. A large portion of parameter space is excluded by the invisible $h$-decay constraint $\operatorname{BR}(h \rightarrow$ inv $)<0.1$, which also forces $\cos \delta \ll 1$. More precisely, the relevant bound is that on the signal strength $\mu$ of the $125 \mathrm{GeV}$ Higgs boson, which constrains a combination of $\sin \theta$ and the invisible decay BR (see, e.g., the discussion in Ref. [35]). Given the fluctuations in the experimental values of $\mu$, we take the resulting constraint on $h \rightarrow$ inv to be $\operatorname{BR}(h \rightarrow$ inv $)<0.1$. The precise value of this bound does not affect our results. In the SU(3) model, the smallness of $\cos \delta$ can be attributed to the Higgs mixing predominantly with one of the triplets, namely, $\phi_{1}$, such 


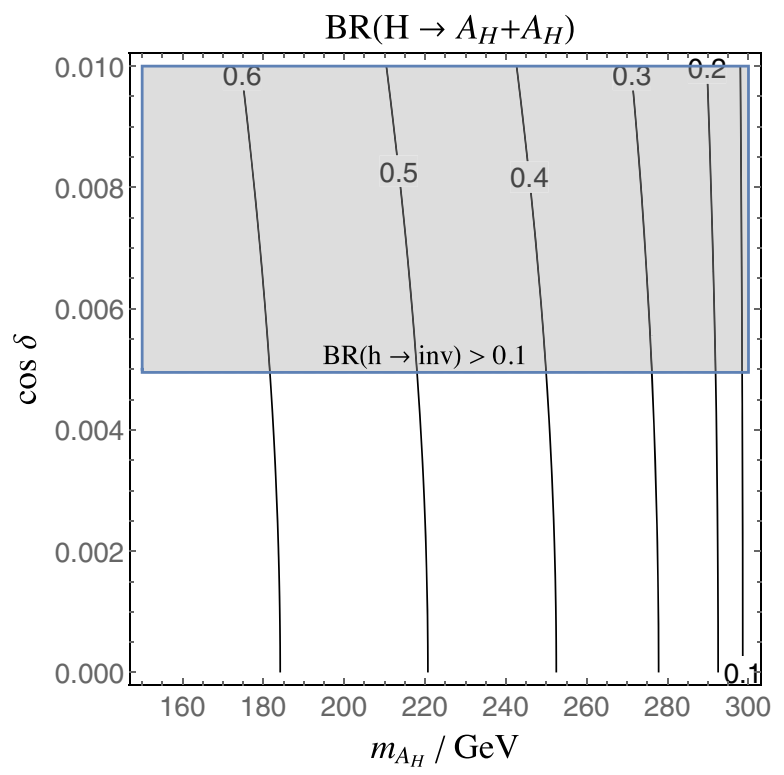

FIG. 2. Branching ratio for the heavy Higgs boson decay to heavier dark bosons $H \rightarrow A_{H} A_{H}$. Here $\sin \theta=0.3, \tilde{g}=2$, $m_{H}=600 \mathrm{GeV}, \quad m_{A_{L}}=10 \mathrm{GeV}$, and $\operatorname{BR}(H \rightarrow h h)=0.2$. The shaded region is excluded by $\mathrm{BR}(h \rightarrow$ inv $)>0.1$.

that $H$ has a large $\phi_{1}$ component (see Table 3 of Ref. [26].) Finally, we note that the chosen values of $\sin \theta$ and $m_{H}$ are consistent with both the LHC and electroweak precision measurements [35,36], although a stronger bound on $\sin \theta$ from the $W$ mass alone has been quoted in Ref. [37].

In this work, we are being agnostic as to the DM production mechanism and do not impose a constraint on the thermal DM annihilation cross section. ${ }^{4}$ On the other hand, the direct DM detection cross section is automatically suppressed: The $h$-mediated contribution is small due to $\cos ^{2} \delta \ll 1$, while the $H$-mediated contribution is suppressed by $\sin ^{2} \theta$ and $m_{H}^{4}$. Thus, in what follows, we may focus on the collider aspects of the model as long as the parameter values are in the ballpark of those considered in this section.

The simplified model can add a layer of complexity by including an extra heavy gauge boson $A_{H}^{\prime}$. Indeed, dark sectors with the symmetry group larger than $\mathrm{SU}(2)$ contain multiple heavy bosons with different masses. The relevant couplings take the form

$$
\mathcal{L} \supset \lambda_{a b i}\left(A_{a}\right)_{\mu}\left(A_{b}\right)^{\mu} \Phi_{i},
$$

where $A_{a}=A_{H}^{\prime}, A_{H}, A_{L}$ and $\Phi_{i}=h, H$. Depending on the couplings $\lambda_{a b i}$ and masses, $H$ may predominantly decay into a pair of $A_{H}^{\prime}$, which then decay into $A_{H}$ and $A_{L}$ with $h$ emission. This can lead, for example, to a $3 h$ and dark matter final state as shown in Fig. 1, right. Such exotic

\footnotetext{
${ }^{4}$ The thermal DM analysis for the SU(3) model has been performed in Ref. [26].
}

processes may provide an additional handle on the dark sector properties.

\section{LHC SEARCH FOR DI-HIGGS BOSON PRODUCTION WITH MISSING ENERGY}

\section{A. $2 b+2 \gamma+E_{T}$ in the final state}

Certain aspects of collider phenomenology of related models have been studied before [6,24,25,38-42] through different production channels. In this work, we focus on the heavy $C P$-even scalar $(H)$ production through gluon fusion, which subsequently decays via the hidden sector vector fields into the $125 \mathrm{GeV}$ Higgs bosons $(h)$ along with two dark matter particles. This can result in various final states depending on the decay mode of the $125 \mathrm{GeV}$ Higgs boson. The dominant decay of the Higgs boson to $b \bar{b}$ has been studied extensively in Ref. [24]. However, such a multi- $b$-jet final state is plagued with hadronic backgrounds, and one needs a very clear understanding of the $W+$ jets and QCD backgrounds at high luminosity in order to isolate the signal events. Using the jet substructure technique to reconstruct the Higgs bosons is an efficient tool for a heavy $H$, while in the mass range of interest to us it is less reliable.

Instead, we explore a cleaner channel where one of the $125 \mathrm{GeV}$ Higgs bosons decays into a pair of photons. The photon identification efficiency is quite good, and, even though the corresponding branching ratio is small, an enhanced cut efficiency makes this channel a significant one. Thus, our signal region consists of two $b$ jets, two photons, and large transverse missing energy. The LHC collaborations have studied the two $b$-jets +2 photons signal region quite extensively in the context of beyond-SM Higgs boson searches [43]. However, this does not include large missing energy which we use as an additional feature to suppress the SM background.

The dominant SM background to the signal arises from the $t \bar{t} h, b \bar{b} h, Z h, \gamma \gamma+$ jets, $t \bar{t} \gamma \gamma, b \bar{b} \gamma \gamma$, and $Z Z \gamma \gamma$ production. In addition, there are contributions from $b \bar{b} \gamma+$ jets and $b \bar{b}+$ jets with the jet(s) being mistagged as photon(s), which can be important due to pileup at the LHC at high luminosity. Although the mistagging probability is small, of the order of $0.1 \%$ [44], the resultant background cross section may not be negligible given the large production cross section for these two processes. We have simulated all of the above processes along with our signal for some representative benchmark points at the $14 \mathrm{TeV}$ LHC. The parton level events have been generated using MadGraph5 $[45,46]$. We use the NNPDF parton distribution function $[47,48]$ for our computation. These events are then passed through PYTHIA8 [49,50] for decay, showering, and hadronization. For processes with additional jets at the parton level, MLM matching [51,52] was performed through the MadGraph5-PYTHIA8 interface. The complete event information is further passed through DELPHES3 [53-55] for 
detector simulation. The jets are constructed via FastJet [56] following the anti-kt [57] algorithm with radius parameter $R=0.4$.

\section{B. Problems with the cut-based analysis}

In our scenario, the two $b$ jets and two photons in the final state arise from on-shell Higgs boson decays. In addition, the final state contains two dark matter particles which contribute to the missing transverse energy. In order to tag the $b$ jets, we have used a tagging efficiency which depends on the $b$ transverse momentum, $0.80 \times \tanh \left(0.003 \times p_{T}\right) \frac{30}{\left(1+0.086 \times p_{T}\right)}$ as provided by the ATLAS Collaboration [58]. We have also considered the possibility of a light jet being mistagged as a $b$ jet. The mistagging probability is largest for the $c$ jets: $0.20 \times \tanh \left(0.02 \times p_{T}\right) \frac{1}{\left(1+0.0034 \times p_{T}\right)}$, and much lower for the other light jets: $0.002+7.3 \times 10^{-6} \times p_{T}$ [58]. In order to tag isolated charged leptons and photons, we impose the requirements $p_{T}>20 \mathrm{GeV}$ and $|\eta|<2.5$, where $\eta$ is the pseudorapidity. We further ensure that all the leptons are well separated among themselves with $\Delta R_{\ell \ell}>0.2$ and also from other particles, $\Delta R_{\ell x}>0.4$, where $x$ can be either photons or jets. Furthermore, the transverse energy deposited by the hadrons within a cone of $\Delta R \leq 0.2$ around the lepton is required to be less than $0.2 \times p_{T}^{\ell}$. Similar $\Delta R$ and hadronic energy deposition criteria are also imposed on the photons for them to be considered isolated. A jetjet separation $\Delta R_{j j}>0.4$ is also imposed. We use the following kinematic cuts to achieve a good signal to background ratio. ${ }^{5}$

(i) $C 1$. - The final state must contain two $b$ jets with $p_{T}^{b}>25 \mathrm{GeV}$ and two photons. Pseudorapidity of both the $b$ jets and the photons must lie within $|\eta|<2.5$. There should be no isolated charged leptons in the final state.

(ii) $C 2$. - The transverse missing energy $\mathscr{E}_{T}$ must be larger than $120 \mathrm{GeV}$. This cut is particularly useful to reduce background contributions from the channels with no direct source of missing energy, namely, $b \bar{b} h, Z h, \gamma \gamma+$ jets, $b \bar{b} \gamma \gamma, b \bar{b} \gamma+$ jets, and $b \bar{b}+$ jets. For all these channels, $E_{T}$ in the final state arises from mismeasurement of the jet transverse momenta. Its distribution is likely to be much softer than that of the channels like $t \bar{t} h, t \bar{t} \gamma \gamma$, and $Z Z \gamma \gamma$ as shown in Fig. 3.

(iii) C3.- Invariant mass of the $b$-jet pair must lie within a $20 \mathrm{GeV}$ window of the $125 \mathrm{GeV}$ Higgs boson, $\left|m_{b \bar{b}}-125 \mathrm{GeV}\right|<20 \mathrm{GeV}$. The background $b$ jets are either hard produced or arise from top quark or $Z$ boson decay. Hence, the invariant mass of the $b$-jet pairs is distributed over a wide range in most cases, whereas for $Z h$ and $Z Z \gamma \gamma$ it peaks around the $Z$

\footnotetext{
${ }^{5}$ Many of these cuts are borrowed from the CMS analysis [43].
}

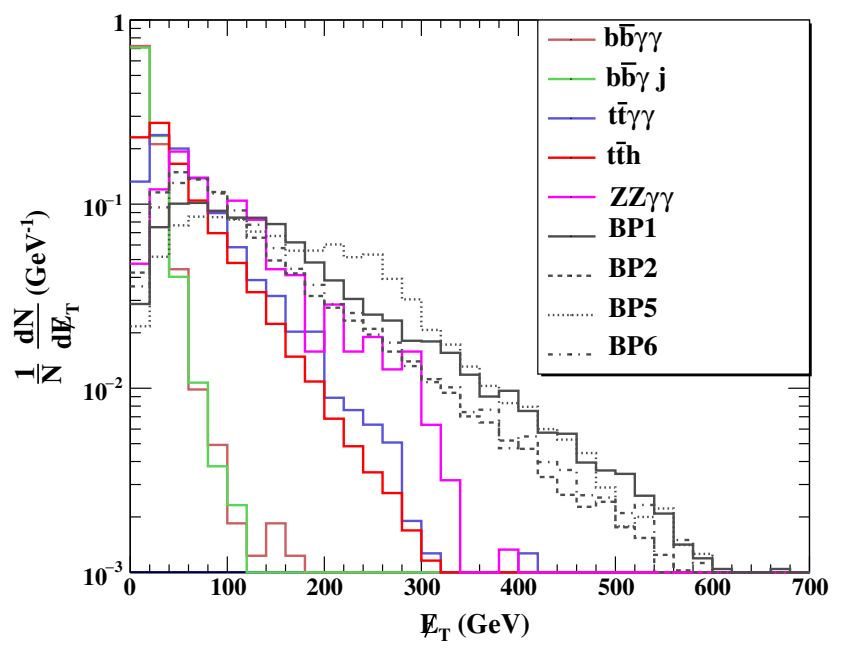

FIG. 3. $E_{T}$ distribution for some of the benchmark points and background processes for the final state $2 b+2 \gamma+E_{T}$.

boson mass as can be seen from Fig. 4. Thus, the cut on the $b \bar{b}$ invariant mass reduces the background contribution drastically.

(iv) C4.-Effective mass $m_{\mathrm{eff}}=\sum_{i} p_{T}^{b_{i}}+\sum_{i} p_{T}^{\gamma_{i}}+\mathscr{E}_{T}$ must be larger than $800 \mathrm{GeV}$. For the signal events, this quantity is normally larger than that for the background channels like $Z h$ and $b \bar{b} h$. This cut also helps to reduce background contributions from soft $b$ jets and photons in processes like $b \bar{b} \gamma \gamma$, $b \bar{b} \gamma+$ jets, and $b \bar{b}+$ jets.

(v) C5.- Invariant mass of the photon pair must lie within a $5 \mathrm{GeV}$ window of the $125 \mathrm{GeV}$ Higgs boson, $\left|m_{\gamma \gamma}-125 \mathrm{GeV}\right|<5 \mathrm{GeV}$. The Higgs boson mass reconstruction from the photon pair is much more precise than that from the $b$ jets due to the better photon momentum resolution, thus allowing for efficient discrimination against many of the

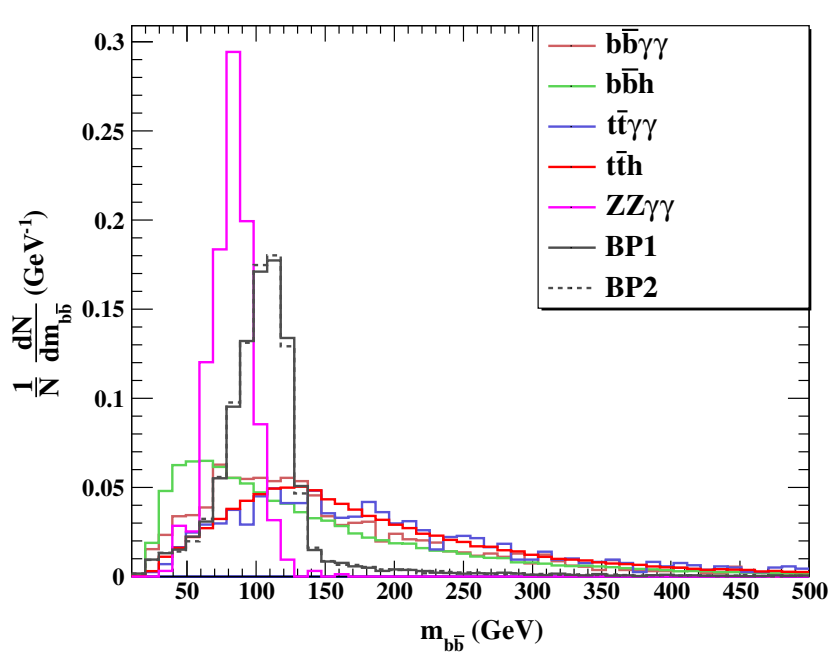

FIG. 4. $m_{b \bar{b}}$ distribution for some of the benchmark points and background processes for the final state $2 b+2 \gamma+\mathscr{E}_{T}$. 


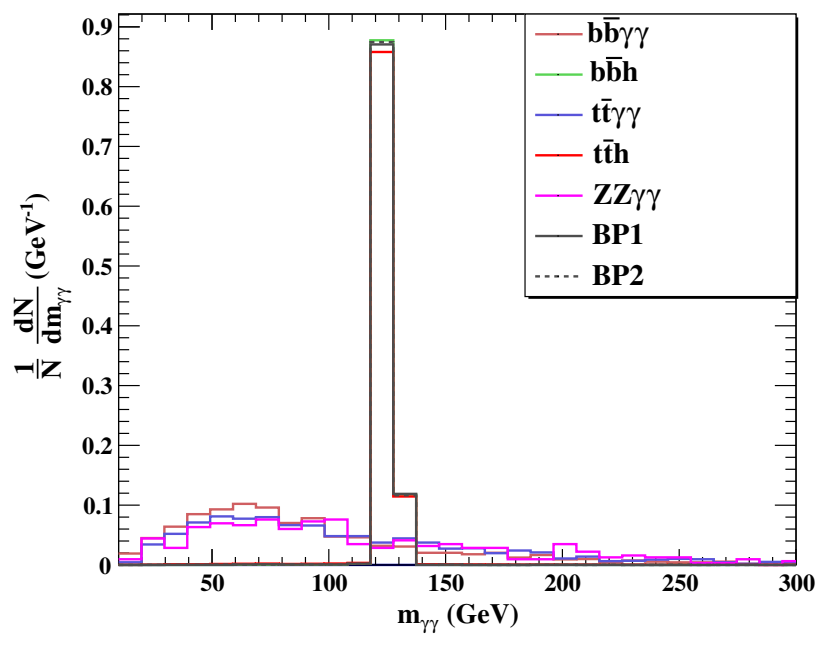

FIG. 5. Distribution of $m_{\gamma \gamma}$ for some sample benchmark points and background processes for the final state $2 b+2 \gamma+E_{T}$.

background channels (except for $t \bar{t} h, b \bar{b} h$, and $Z h$ ). The corresponding kinematic distribution for some of the signal and background processes is shown in Fig. 5.

(vi) C6.--Angular separation between the $b \bar{b}$ pair and the photon pair must be large, $\Delta \phi(b \bar{b}, \gamma \gamma)>2.0$. Since the two Higgs bosons are radiated from two different legs in the production process, it is expected that the resultant $b$-jet pair and the $\gamma$ pair will be well separated in the final state. This is not the case for some of the background processes, especially those where all the $b$ jets and photons are hard produced, like $b \bar{b} \gamma \gamma$, etc. The two $b$ jets can also arise from decays of two different mother particles, as in $t \bar{t} \gamma \gamma$, in which case the combined $b \bar{b}$ system may not be well separated from the $\gamma \gamma$ system.

(vii) $C 7 . \frac{p_{T}^{\gamma_{1}}}{m_{\gamma \gamma}}>\frac{1}{3}$ and $\frac{p_{T}^{\gamma_{2}}}{m_{\gamma \gamma}}>\frac{1}{4}$. This eliminates some of the distortions in the low end of the $m_{\gamma \gamma}$ distribution and reduces the continuum background.

In Tables II and III, we show our results obtained from the cut-based analysis. The signal strength depends strongly on $m_{H}, \sin \theta$, and $\operatorname{BR}\left(H \rightarrow A_{H} A_{H}\right)$. For these we choose the benchmark values $\sin \theta=0.3$ and $\operatorname{BR}\left(H \rightarrow A_{H} A_{H}\right)=1.0$ and 0.7 in order to maximize the event rate, while $m_{H}$ is taken to be $500 \mathrm{GeV}$ and above. For lower $m_{H}$, the existing LHC data already constrain the model as we show in the subsequent section. Hence, $m_{H} \geq 500 \mathrm{GeV}$ represents the range of interest for the future LHC runs, as long as $\sin \theta$ and $\operatorname{BR}\left(H \rightarrow A_{H} A_{H}\right)$ are relatively large. To paint a more complete picture, in Sec. 3.4. 1 we discuss the LHC reach in terms of $m_{H}$ leaving $\mathrm{BR}\left(H \rightarrow A_{H} A_{H}\right)$ as a free parameter.

Cuts $\mathrm{C} 2, \mathrm{C} 3$, and $\mathrm{C} 5$ prove to be the most effective ones in reducing the background. Clearly, the combined impact of the chosen cuts is adequate for our purposes, yet they also decrease the signal cross section to the extent that any possible signal could be observed only at high-luminosity
TABLE II. The SM background channels along with their cross sections before and after the cuts. Numerically negligible results are not shown. Here we have used a flat rate of $0.1 \%$ for the probability of a jet being mistagged as a photon.

\begin{tabular}{lcc}
\hline \hline Channels & $\begin{array}{c}\text { Production cross } \\
\text { section (fb) }\end{array}$ & $\begin{array}{c}\text { Cross section } \\
\text { after cuts (fb) }\end{array}$ \\
\hline$t \bar{t} h(h \rightarrow \gamma \gamma)$ & 0.673 & $2.2 \times 10^{-4}$ \\
$b \bar{b} h(h \rightarrow \gamma \gamma)$ & 0.164 & $6.4 \times 10^{-6}$ \\
$Z h(Z \rightarrow b \bar{b}, h \rightarrow \gamma \gamma)$ & 0.141 & $\ldots$ \\
$\gamma \gamma+$ jets & 61660.7 & $\ldots$ \\
$t \bar{t} \gamma \gamma$ & 2457.6 & $2.4 \times 10^{-3}$ \\
$b \bar{b} \gamma \gamma$ & 5144.41 & $\ldots$ \\
$Z Z(\rightarrow \nu \bar{\nu} b \bar{b})+$ photons & 2.106 & $\ldots$ \\
$b \bar{b} \gamma+$ jets & $9.76 \times 10^{6}$ & $\ldots$ \\
$b \bar{b}+$ jets & $7.74 \times 10^{9}$ & \\
\hline \hline
\end{tabular}

LHC. The $14 \mathrm{TeV}$ LHC is projected to accumulate an integrated luminosity of $3000 \mathrm{fb}^{-1}$, which appears insufficient to obtain even $3 \sigma$ statistical significance in most of the parameter space. ${ }^{6}$ We have also tried to soften the cuts to increase the signal rate, but that eventually results in a worse signal to background ratio. The best case scenario here is BP1, for which the significance factor at $3000 \mathrm{fb}^{-1}$ is $\sim 2.5 \sigma$ if we assume $\sigma_{B}=0$. Therefore, our conclusion is that imposing rectangular cuts does not lead to good discovery prospects.

\section{Multivariate analysis}

Evidently, the cut-based analysis is not sensitive enough to probe the present scenario at the $14 \mathrm{TeV}$ LHC even at high luminosity. Thus, we next explore the possibility of improving the analysis with machine learning techniques, namely, the gradient boosted decision trees (BDT) [59]. This method of data analysis is being used quite extensively in LHC searches to good effect [24,60-64]. We have chosen the XGBoost [59] toolkit for the gradient boosting analysis. Below, we list the kinematic variables used in the decision trees (cf. [43]).

(1) Number of $b$ jets, $N_{b}$.

(2) Number of photons, $N_{\gamma}$.

(3) Transverse momentum of the hardest $b$ jet, $p_{T}^{b_{1}}$.

(4) Transverse momentum of the second hardest $b$ jet, $p_{T}^{b_{2}}$.

(5) Transverse momentum of the hardest photon, $p_{T}^{\gamma_{1}}$.

(6) Transverse momentum of the second hardest photon, $p_{T}^{\gamma_{2}}$.

(7) Missing transverse energy $E_{T}$.

(8) Invariant mass of the hardest $b$ pair, $M_{\mathrm{inv}}^{b \bar{b}}$.

\footnotetext{
${ }^{6} \mathrm{We}$ use the following definition of statistical significance: $\mathcal{S}=S / \sqrt{S+B+\sigma_{B}^{2}}$, where $S$ and $B$ are the number of signal and background events, respectively, and the background uncertainty is taken to be negligibly small, $\sigma_{B} \ll B$.
} 
TABLE III. The signal cross section $\sigma\left(p p \rightarrow H \rightarrow A_{H} A_{H} \rightarrow h A_{L} h A_{L} \rightarrow b \bar{b} \gamma \gamma A_{L} A_{L}\right)$ before and after the cuts. For all the benchmark points, $\sin \theta=0.3, \operatorname{BR}\left(H \rightarrow A_{H} A_{H}\right)=1.0$ or 0.7 (in parentheses), and $\operatorname{BR}\left(A_{H} \rightarrow A_{L} h\right)=1.0$.

\begin{tabular}{lcc}
\hline \hline Benchmark points & $\begin{array}{c}\text { Production cross } \\
\text { section }(\mathrm{fb})\end{array}$ & $\begin{array}{c}\text { Cross section after } \\
\text { cuts }(\mathrm{fb})\end{array}$ \\
\hline $\mathrm{BP} 1:$ & & 0.640 \\
$m_{H}=500 \mathrm{GeV}, m_{A_{H}}=240 \mathrm{GeV}, m_{A_{L}}=10 \mathrm{GeV}$ & $(0.448)$ & $3.66 \times 10^{-3}$ \\
$\mathrm{BP}$ : & & $\left(2.56 \times 10^{-3}\right)$ \\
$m_{H}=500 \mathrm{GeV}, m_{A_{H}}=240 \mathrm{GeV}, m_{A_{L}}=100 \mathrm{GeV}$ & $3.05 \times 10^{-3}$ \\
$\mathrm{BP}$ : & & $\left(2.14 \times 10^{-3}\right)$ \\
$m_{H}=600 \mathrm{GeV}, m_{A_{H}}=280 \mathrm{GeV}, m_{A_{L}}=10 \mathrm{GeV}$ & 0.286 & $9.25 \times 10^{-4}$ \\
BP4: & $(0.200)$ & $\left(6.48 \times 10^{-4}\right)$ \\
$m_{H}=600 \mathrm{GeV}, m_{A_{H}}=280 \mathrm{GeV}, m_{A_{L}}=150 \mathrm{GeV}$ & & $8.95 \times 10^{-4}$ \\
BP5: & & $\left(6.27 \times 10^{-4}\right)$ \\
$m_{H}=700 \mathrm{GeV}, m_{A_{H}}=340 \mathrm{GeV}, m_{A_{L}}=10 \mathrm{GeV}$ & 0.133 & $4.16 \times 10^{-4}$ \\
BP6: & $(0.093)$ & $\left(2.91 \times 10^{-4}\right)$ \\
$m_{H}=700 \mathrm{GeV}, m_{A_{H}}=340 \mathrm{GeV}, m_{A_{L}}=200 \mathrm{GeV}$ & & $3.33 \times 10^{-4}$ \\
& & $\left(2.33 \times 10^{-4}\right)$ \\
\hline \hline
\end{tabular}

(9) Invariant mass of the hardest photon pair, $M_{\mathrm{inv}}^{\gamma \gamma}$.

(10) Effective mass $M_{\text {eff }}$, defined as the scalar sum of the transverse momenta of the jets, photons, and $E_{T}$.

(11) Angular separation between the $b \bar{b}$ pair and the photon pair, $\Delta \phi(b \bar{b}, \gamma \gamma)$.

(12) Ratio of the hardest photon $p_{T}$ and diphoton invariant mass, $R_{1}=\frac{p_{T}^{\gamma_{1}}}{M_{\text {inv }}^{\gamma \gamma}}$.

(13) Ratio of the second hardest photon $p_{T}$ and diphoton invariant mass, $R_{2}=\frac{p_{T}^{\gamma_{2}}}{M_{\text {inv }}^{\gamma \gamma}}$.

We have chosen 1000 trees, maximum depth 4, and learning rate 0.01 for our analysis. We combine data on the above kinematic variables for our signal events with all the background events in one data file. All the events are required to have at least two $b$ jets $\left(p_{T}^{b}>25 \mathrm{GeV}\right)$, at least two photons $\left(p_{T}^{\gamma}>20 \mathrm{GeV}\right)$, and no charged leptons with $p_{T}>20 \mathrm{GeV}$. We have combined the background events after properly weighting them according to their cross sections subject to these cuts. As a result, more importance is given to the dominant backgrounds while training the data. We also make sure that there are enough signal events to match the total weight of the background events. We take $80 \%$ of our data for training and $20 \%$ for testing. Each of our data samples typically contains values of the kinematic variables corresponding to $\sim 10^{6}$ events. These include the final states subject to our conditions on $b$ jets, photons, and charged leptons as discussed above. In order to obtain this dataset, we have generated $\sim 10^{7}$ Monte Carlo events for the backgrounds and $\sim 10^{6}$ events for the signal benchmark points.

\section{Results}

Figure 6 shows results of our multivariate analysis for benchmark point BP1. The BDT classifier response shows clear distinction between the signal and the background.
We find that, among the kinematic variables listed above, $M_{\text {inv }}^{b \bar{b}}$ and $E_{T}$ are the two most important discriminators followed by $M_{\text {inv }}^{\gamma \gamma}$. The right panel in Fig. 6 shows the receiver operating characteristic (ROC) curve for BP1. The $x$ axis (efficiency) indicates the fraction of identified signal events after imposing the BDT classifier, while the $y$ axis (purity) indicates the ratio of identified signal events to the total number of identified events (signal plus background) after imposing the classifier. The area under the curve (AUC) is a good indicator of the BDT performance. For this benchmark point, $\mathrm{AUC}=0.90$.

Our multivariate analysis results are quite promising and prove to be a significant improvement over those for the cut-based analysis. The AUC indicator is close to 0.9 for all the benchmark points, which shows that the BDT classifier is efficient in distinguishing the signal from the background in all considered cases. In Table IV, we present the AUC for the benchmark points along with the required luminosity to achieve a signal significance of $3 \sigma$.

This approach allows one to constrain the low $H$-mass window already with the existing LHC data. The integrated luminosity required to achieve the $3 \sigma$ significance for $m_{H}=450 \mathrm{GeV}, m_{A_{H}}=150 \mathrm{GeV}, \sin \theta=0.3$, and $\operatorname{BR}\left(H \rightarrow A_{H} A_{H}\right)=1.0,0.7$ is 72 and $114 \mathrm{fb}^{-1}$, respectively. Thus, the current set of $139 \mathrm{fb}^{-1}$ of data collected should be sufficient to exclude $m_{H} \leq 450 \mathrm{GeV}$ for these parameter values, although the $2 b+2 \gamma+E_{T}$ channel has not yet been analyzed by ATLAS and CMS. This justifies our choice of the $m_{H}$ benchmark values of $500 \mathrm{GeV}$ and above for the future LHC runs.

From our BDT analysis, we conclude that nine out of the 13 kinematic variables used are essential to obtain the sensitivity indicated in Table IV. The four less essential variables are $N_{b}, N_{\gamma}, R_{1}$, and $R_{2}$. Among these, $N_{b}$ and $N_{\gamma}$ 

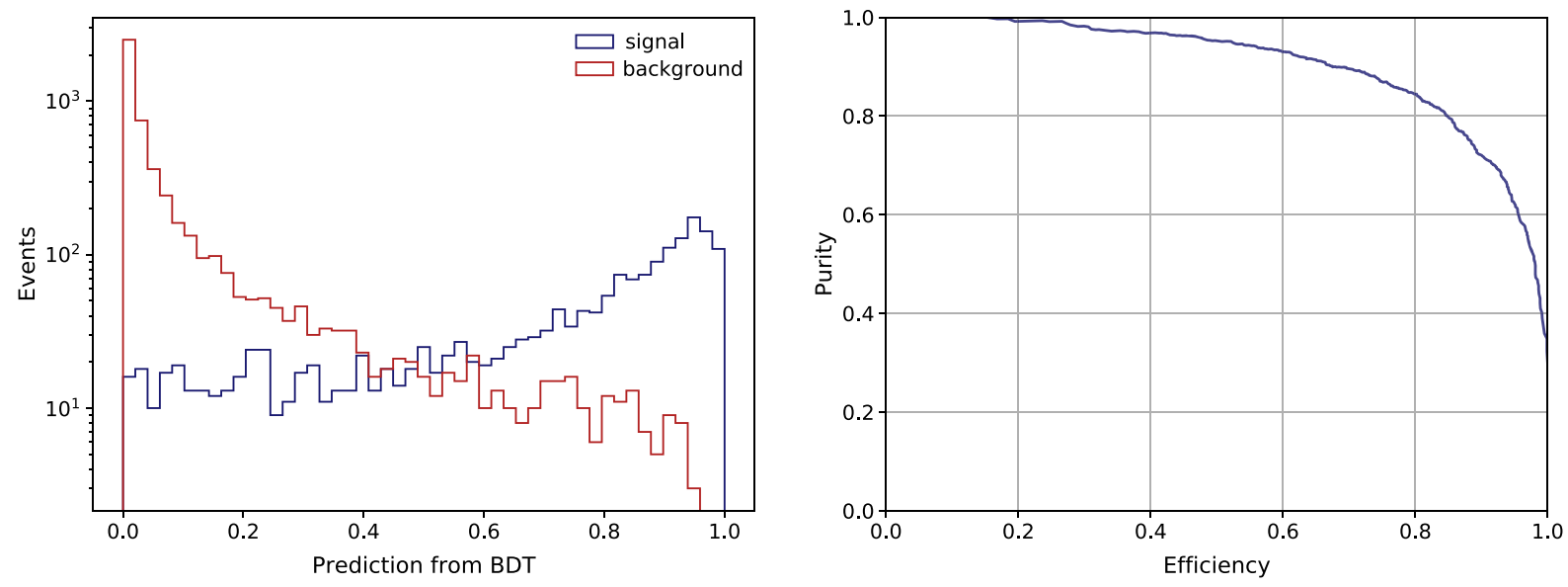

FIG. 6. Example of the BDT classifier response for BP1 $\left(m_{H}=500 \mathrm{GeV}, m_{A_{H}}=240 \mathrm{GeV}, m_{A_{L}}=10 \mathrm{GeV}\right)$ in differentiating the signal and background events. The ROC curve in the right panel indicates the efficiency of the algorithm.

are rendered inconsequential by the criteria we have set to select the final states, which ensure that all the signal and background events already have at least one pair of $b$ jets and photons. Some of the important kinematic variables, specifically $p_{T}^{\gamma_{1}}, p_{T}^{\gamma_{2}}$, and $M_{\text {inv }}^{\gamma \gamma}$, ensure that $R_{1}$ and $R_{2}$ are not essential either. With the remaining nine variables, we have performed a tenfold cross validation check in order to assess the robustness of our analysis. We have randomly split our dataset in train and test sets of similar size and computed the signal significance for ten such different combinations. In Table $\mathrm{V}$, we present the resulting signal significance factors obtained at an integrated luminosity of $1 \mathrm{fb}^{-1}$ along with the corresponding mean and the variance

TABLE IV. The area under the ROC curve for the benchmark points (see Table III) along with the required luminosity to achieve a $3 \sigma$ signal significance in the $2 b+2 \gamma+E_{T}$ channel at the $14 \mathrm{TeV}$ LHC assuming $\operatorname{BR}\left(H \rightarrow A_{H} A_{H}\right)=1$ or 0.7 (in parentheses).

\begin{tabular}{lcc}
\hline \hline Benchmark points & $\begin{array}{c}\text { Area under } \\
\text { the ROC curve }\end{array}$ & $\begin{array}{c}\text { Required luminosity } \\
\left(\mathrm{fb}^{-1}\right)\end{array}$ \\
\hline BP1 & 0.89 & $300(505)$ \\
BP2 & 0.87 & $707(1264)$ \\
BP3 & 0.91 & $870(1433)$ \\
BP4 & 0.88 & $2255(4174)$ \\
BP5 & 0.91 & $3911(7381)$ \\
BP6 & 0.88 & $5995(11469)$ \\
\hline \hline
\end{tabular}

TABLE V. Significance factors, their mean, and their variance obtained in our tenfold validation procedure for $2 b+2 \gamma+E_{T}$ at $\mathrm{BP} 1$. The integrated luminosity is taken to be $1 \mathrm{fb}^{-1}$.

\begin{tabular}{lcc}
\hline \hline Significance factors & Mean & Variance \\
\hline $0.171,0.174,0.172,0.168,0.175$, & 0.1726 & $3.0 \times 10^{-3}$ \\
$0.171,0.176,0.178,0.169,0.172$ & & \\
\hline \hline
\end{tabular}

for the benchmark point BP1. The variance is clearly quite small, indicating the robustness of our results.

\section{Multivariate analysis for $2 b$ jets $+2 \mathscr{C}+\mathscr{E}_{T}$ in the final state}

Another promising channel for the di-Higgs boson and dark matter search is provided by the $\bar{b} b$ and $W W^{*}$ decay modes of the Higgs bosons. Tagging on leptonic decays of the $W$ 's, one obtains a clean final state with $2 b$ jets $+2 \ell+t_{T}$. Here we take $\ell=e, \mu$. The challenge is to suppress the $t \bar{t}+$ jets background, which has a much larger cross section. Further background contributions come from the $t \bar{t} V, t \bar{t} h, V V V$, and $V V+$ jets $\left(V \equiv W^{ \pm}, Z\right)$ channels. We perform a multivariate analysis of our signal with the following kinematic variables (cf. [65]):

(1) Number of $b$ jets, $N_{b}$.

(2) Number of charged leptons ( $e$ or $\mu$ ), $N_{\ell}$.

(3) Number of non- $b$-tagged jets, $N_{j}$.

(4) Transverse momentum of the hardest $b$ jet, $p_{T}^{b_{1}}$.

(5) Transverse momentum of the second hardest $b$ jet, $p_{T}^{b_{2}}$

(6) Transverse momentum of the hardest charged lepton, $p_{T}^{\ell_{1}}$.

(7) Transverse momentum of the second hardest charged lepton, $p_{T}^{\ell_{2}}$.

(8) Missing transverse energy $\mathscr{E}_{T}$.

(9) Effective mass defined as a scalar sum of the transverse momenta of the $b$ jets, leptons, and $E_{T}$.

(10) Invariant mass of the $b$-jet pair, $m_{\text {inv }}^{b \bar{b}}$.

(11) Invariant mass of the lepton pair, $m_{\text {inv }}^{\ell \bar{\ell}}$.

(12) Transverse momentum of the dilepton system, $p_{T}^{\ell \bar{\ell}}$.

(13) Transverse momentum of the $b$-jet pair, $p_{T}^{b \bar{b}}$.

(14) Separation between the two charged leptons, $\Delta R^{\ell \bar{\ell}}$.

(15) Separation between the two $b$ jets, $\Delta R^{b \bar{b}}$.

(16) Azimuthal separation between the $b$-jet pair and the dilepton system, $\Delta \phi(b \bar{b}, \ell \bar{\ell})$. 
TABLE VI. The area under the ROC curve for the benchmark points (see Table III) along with the required integrated luminosity to achieve a $3 \sigma$ signal significance in the $2 b+2 \ell+E_{T}$ at the $14 \mathrm{TeV}$ LHC assuming $\operatorname{BR}\left(H \rightarrow A_{H} A_{H}\right)=1$ or 0.7 (in parentheses). BP5 and BP6 require very large integrated luminosity and, thus, are not shown.

\begin{tabular}{lcc}
\hline \hline Benchmark points & $\begin{array}{c}\text { Area under the } \\
\text { ROC curve }\end{array}$ & $\begin{array}{c}\text { Required luminosity } \\
\left(\mathrm{fb}^{-1}\right)\end{array}$ \\
\hline BP1 & 0.88 & $463(900)$ \\
BP2 & 0.87 & $819(1614)$ \\
BP3 & 0.85 & $2900(5804)$ \\
BP4 & 0.83 & $11468(23192)$ \\
\hline \hline
\end{tabular}

\section{Results}

We collect information on these kinematic variables subject to the following preliminary cuts: The final state is required to have at least two $b$ jets with $p_{T}>25 \mathrm{GeV}$, at least two charged leptons with $p_{T}>20 \mathrm{GeV}$, and $E_{T}>$ $50 \mathrm{GeV}$. The final results are summarized in Table VI. In Fig. 7, we present the required integrated luminosity for a $3 \sigma$ signal significance in both channels.

Apart from $N_{b}$ and $N_{\ell}$, all the kinematic variables are essential with $m_{\text {inv }}^{b \bar{b}}, \mathscr{E}_{T}, m_{\text {inv }}^{\ell \bar{\ell}}$, and $\Delta R^{b \bar{b}}$ being the most important ones. We have performed a tenfold cross validation check for this analysis as well following the prescription of Sec. III C 1. In Table VII, we present the resulting ten signal significance factors obtained at an integrated luminosity of $1 \mathrm{fb}^{-1}$ along with the corresponding mean and the variance for the benchmark point BP1. The variance is sufficiently small indicating robustness of our results.

The results can also presented in terms of the exclusion limits on the model. In particular, the branching ratio for the decay $H \rightarrow A_{H} A_{H}$ can be severely constrained with $3 \mathrm{ab}^{-1}$ of data. Figure 8 displays the corresponding bound for a representative set of parameters, namely, $m_{A_{H}}=150 \mathrm{GeV}$, $m_{A_{L}}=10 \mathrm{GeV}$, and $\sin \theta=0.2,0.3$. The constraint can be as strong as $\operatorname{BR}\left(H \rightarrow A_{H} A_{H}\right)<7 \%$ for $m_{H}<400 \mathrm{GeV}$. Substantial values of $\operatorname{BR}\left(H \rightarrow A_{H} A_{H}\right)$ can be probed up to
TABLE VII. Significance factors, their mean, and their variance obtained in our tenfold validation procedure for $2 b+2 \ell+E_{T}$ at $\mathrm{BP} 1$. The integrated luminosity is taken to be $1 \mathrm{fb}^{-1}$.

\begin{tabular}{lcc}
\hline \hline Significance factors & Mean & Variance \\
\hline $0.143,0.144,0.140,0.138,0.143,0.137$, & 0.1394 & $2.8 \times 10^{-3}$ \\
$0.136,0.136,0.139,0.138$ & & \\
\hline \hline
\end{tabular}

$m_{H} \sim 600-700 \mathrm{GeV}$. We also see that the $2 b+2 \gamma+E_{T}$ channel performs slightly better than $2 b+2 \ell+E_{T}$ does.

So far, we have presented our results assuming $\sigma_{B} \simeq 0$. It is very difficult to forecast the future background uncertainty, so we have to resort to simple estimates. Let us assume the background uncertainty to be $20 \%$ of the total background, i.e., $\sigma_{B}=0.2 \times B$. Consequently, $\sigma_{B}^{2}$ can be very large for signal regions with a large background cross section. The $2 b+2 \ell+E_{T}$ signal is thus affected quite severely, since $t \bar{t}+$ jets is a direct background to this final state and it comes with a large irreducible cross section. We find that, at $3000 \mathrm{fb}^{-1}$ luminosity with the parameter choices shown in Fig. 8, the signal significance for the $2 b+2 \gamma+E_{T}$ final state reduces by a factor of $\sim 2$ when $\sigma_{B}=0.2 \times B$ compared to the case with $\sigma_{B}=0$, whereas for $2 b+2 \ell+E_{T}$ it reduces by $\sim 10$. Since at such high luminosity $\mathcal{S}=S / \sqrt{S+B+\sigma_{B}^{2}} \simeq S / \sqrt{B+\sigma_{B}^{2}}$, the required $\operatorname{BR}\left(H \rightarrow A_{H} A_{H}\right)$ in the $2 b+2 \gamma+E_{T}$ and $2 b+2 \ell+E_{T}$ final states have to be scaled up by factors of 2 and 10 , respectively. The scale factor remains the same for the different choices of $\sin \theta$, since the kinematics of the final state are not affected.

\section{LHC SEARCH FOR A TRI-HIGGS BOSON AND DARK MATTER FINAL STATE}

\section{A. Multivariate analysis for $\geq 3 b$-jets $+2 \ell+E_{T}$ in the final state}

In the presence of additional heavy particles in the dark sector, more exotic final states can be produced. In particular, cascade decays can lead to three or more Higgs

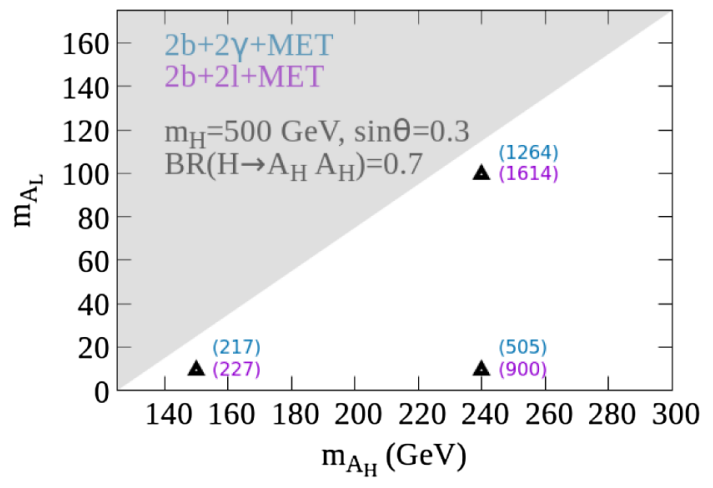

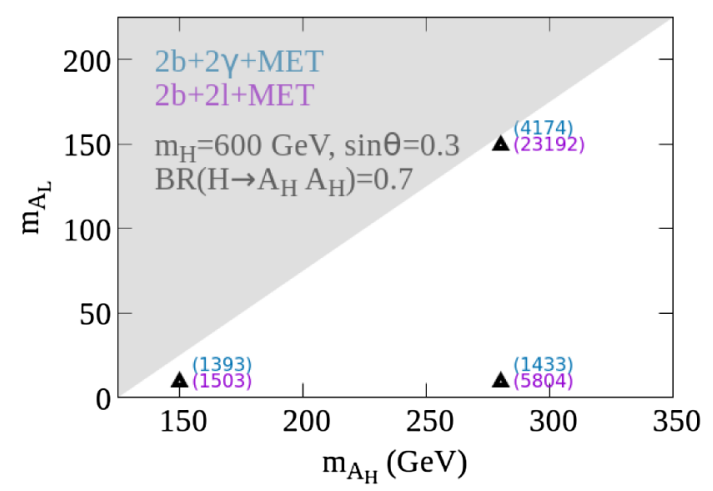

FIG. 7. Integrated luminosity required for a $3 \sigma$ signal significance in the $2 b+2 \gamma+E_{T}$ and $2 b+2 \ell+E_{T}$ channels at 14 TeV LHC. 


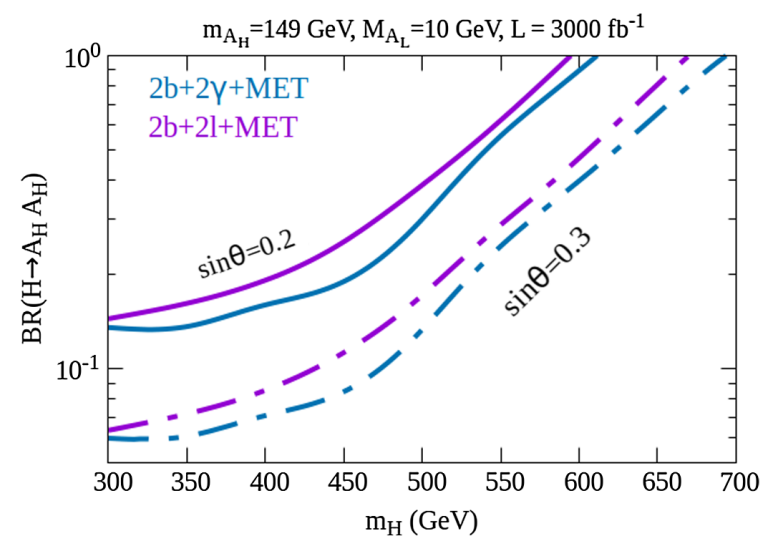

FIG. 8. $3 \sigma$ sensitivity of the $2 b+2 \gamma+E_{T}$ and $2 b+2 \ell+E_{T}$ channels to $\operatorname{BR}\left(H \rightarrow A_{H} A_{H}\right)$ at $14 \mathrm{TeV}$ LHC with integrated luminosity of $3000 \mathrm{fb}^{-1}$. The dashed (solid) line corresponds to $\sin \theta=0.3(\sin \theta=0.2)$.

bosons $h$ in the final state. For example, the heavy Higgs boson $H$ can produce a pair of heavy $A_{H}^{\prime}$, which, in turn, decay via two different decay modes $A_{H}^{\prime} \rightarrow A_{H} h$ and $A_{H}^{\prime} \rightarrow A_{L} h$. Subsequently, $A_{H}$ decays into $A_{L}$ and $h$, thereby generating a multi-Higgs boson final state. The channel with four Higgs bosons suffers from severe kinematic suppression, while the tri-Higgs boson one could potentially be interesting.

Multi-Higgs boson production can be efficient if the decays happen on shell. This implies that $A_{H}^{\prime}$ must be heavier than $250 \mathrm{GeV}$ and, thus, $m_{H}>500 \mathrm{GeV}$. Given a tri-Higgs boson final state, there are a number of options to consider for Higgs boson decay. If all three decay into $\bar{b} b$ pairs, the signal extraction is marred by a large QCD multijet background and possible misidentification of light flavor jets as $b$ jets. For the current study, we choose instead two $\bar{b} b$ pairs and leptonically decaying $W W^{*}$ as our final state. It has the advantage of being relatively clean, and, in addition, we may recycle many of the background calculations done in the previous section. We perform a multivariate analysis with the following kinematic variables:

(1) Number of $b$ jets, $N_{b}$.

(2) Number of charged leptons ( $e$ or $\mu$ ), $N_{\ell}$.

(3) Number of non- $b$-tagged jets, $N_{j}$.

(4) Transverse momentum of the hardest $b$ jet, $p_{T}^{b_{1}}$, involved in a $b$-jet pair with $m_{\text {inv }}^{b \bar{b}}$ closest to $125 \mathrm{GeV}$.

(5) Transverse momentum of the second hardest $b$ jet, $p_{T}^{b_{2}}$, involved in a $b$-jet pair with $m_{\text {inv }}^{b \bar{b}}$ closest to $125 \mathrm{GeV}$.

(6) Transverse momentum of the hardest charged lepton, $p_{T}^{\ell_{1}}$.

(7) Transverse momentum of the second hardest charged lepton, $p_{T}^{\ell_{2}}$.

(8) Missing transverse energy $\mathscr{E}_{T}$.

(9) Effective mass, defined as a scalar sum of the transverse momenta of the $b$ jets, leptons, and $E_{T}$.
(10) Invariant mass of the $b$-jet pair, $m_{\text {inv }}^{b_{1} b_{2}}$.

(11) Invariant mass of the lepton pair, $m_{\mathrm{inv}}^{\ell \bar{\ell}}$.

(12) Transverse momentum of the dilepton system, $p_{T}^{\ell \bar{\ell}}$.

(13) Transverse momentum of the $b$-jet pair, $p_{T}^{b_{1} b_{2}}$.

(14) Separation between the two charged leptons, $\Delta R^{\ell \bar{\ell}}$.

(15) Separation between the two $b$ jets, $\Delta R^{b_{1} b_{2}}$.

(16) Azimuthal separation between the $b$-jet pair and the dilepton system, $\Delta \phi\left(b_{1} b_{2}, \ell \bar{\ell}\right)$.

We collect information on these kinematic variables imposing the following preliminary cuts: The final state is required to have at least three $b$ jets with $p_{T}>25 \mathrm{GeV}$ and at least two charged leptons with $p_{T}>20 \mathrm{GeV}$. We construct all possible $b$-jet pairs in the final state, compute the corresponding invariant masses, and then identify the pair that has $m_{\text {inv }}^{b \vec{b}}$ closest to $125 \mathrm{GeV}$. We call the harder $b$ jet in this pair $b_{1}$, and the other one is identified as $b_{2}$.

Note that we are not using the third $b$-jet kinematics directly in our multivariate analysis. Including it adds a few more variables to the list: $p_{b_{3}}^{T}, \Delta \phi\left(b_{3}, \ell \bar{\ell}\right)$, and $\Delta \phi\left(b_{1} b_{2}, b_{3}\right)$. However, we have verified that these do not improve our results.

The sensitivity of this signal region is weaker compared to the previous two. Although the BDT classifier is quite efficient in isolating the signal events, the few remaining background events have a large enough cross section to suppress the signal significance. This is due to the small signal cross section to start with, which is further reduced by detector simulations and applying the BDT classifier.

To give an example, consider the parameter set $m_{H}=$ $600 \mathrm{GeV}, m_{A_{H}^{\prime}}=290 \mathrm{GeV}, m_{A_{H}}=150 \mathrm{GeV}$, and $m_{A_{L}}=$ $20 \mathrm{GeV}$. The production cross section for the required final state is $0.107 \mathrm{fb}$. Optimizing the signal to background ratio using our multivariate analysis, we find that the $1 \sigma$ signal significance is achieved with $3000 \mathrm{fb}^{-1}$ integrated luminosity, while that at the $2 \sigma$ level requires $12000 \mathrm{fb}^{-1}$. Clearly, this is beyond the reach of the LHC.

Although our result for the $3 b$ jets $+2 \ell+E_{T}$ channel is negative, a fully $b$-jet $+E_{T}^{\prime}$ final state may be more promising. It requires a dedicated analysis which we reserve for future work.

\section{SUMMARY AND CONCLUSIONS}

In this work, we have considered dark sectors with spontaneously broken gauge symmetries, where dark cascade decays naturally lead to multi-Higgs boson final states with missing energy. We have introduced a simplified model which captures the main features of realistic hidden sectors containing dark matter as well as further heavier states.

The focus of this work is two and three Higgs boson final states which subsequently decay into $\bar{b} b, \gamma \gamma$, and $W W$. Using multivariate analysis with boosted decision trees, we find that the $2 b+2 \gamma+E_{T}$ and $2 b+2 \ell+E_{T}$ channels are promising in the context of $14 \mathrm{TeV}$ LHC with $3 \mathrm{ab}^{-1}$ 
integrated luminosity. In particular, light dark matter $A_{L}$ with mass $\lesssim 100 \mathrm{GeV}$ can be probed efficiently for the dark Higgs boson $(H)$ mass below $700 \mathrm{GeV}$ and its mixing angle with the SM Higgs boson $\sin \theta \sim 0.2-0.3$. In this region, $3 \sigma$ and higher signal significance can be achieved. The result can also be translated into a bound on the dark Higgs boson decay into the heavier partners of dark matter $A_{H}$, with sensitivity to $\operatorname{BR}\left(H \rightarrow A_{H} A_{H}\right)$ reaching $6 \%$ in the best case scenario.

The three Higgs boson final state, on the other hand, appears far less promising, at least for the decay channels considered. Fully hadronic Higgs boson decays may change this situation but require a dedicated background study and detection simulation.

\section{ACKNOWLEDGMENTS}

M.F. is supported by the National Research Foundation of South Africa-The World Academy of Sciences (NRF-TWAS) Grant No. 110790, Reference No. SFH170609238739. C. G. is supported by the European Research Council grant NEO-NAT.
[1] V. Silveira and A. Zee, Phys. Lett. 161B, 136 (1985).

[2] R. M. Schabinger and J. D. Wells, Phys. Rev. D 72, 093007 (2005).

[3] B. Patt and F. Wilczek, arXiv:hep-ph/0605188.

[4] B. Di Micco et al., arXiv:1910.00012.

[5] K. T. Matchev and S. D. Thomas, Phys. Rev. D 62, 077702 (2000).

[6] Z. Kang, P. Ko, and J. Li, Phys. Rev. Lett. 116, 131801 (2016).

[7] S. M. Etesami and M. Mohammadi Najafabadi, Phys. Rev. D 92, 073013 (2015).

[8] A. Papaefstathiou and K. Sakurai, J. High Energy Phys. 02 (2016) 006.

[9] X. F. Han, L. Wang, and J. M. Yang, Mod. Phys. Lett. A 31, 1650178 (2016).

[10] Z. Kang, P. Ko, and J. Li, Phys. Rev. D 93, 075037 (2016).

[11] S. Biswas, E. J. Chun, and P. Sharma, J. High Energy Phys. 12 (2016) 062.

[12] I. Brivio, M. B. Gavela, L. Merlo, K. Mimasu, J. M. No, R. del Rey, and V. Sanz, Eur. Phys. J. C 77, 572 (2017).

[13] E. Arganda, J. L. Díaz-Cruz, N. Mileo, R. A. Morales, and A. Szynkman, Nucl. Phys. B929, 171 (2018).

[14] C. R. Chen, Y.X. Lin, H. C. Wu, and J. Yue, arXiv: 1804.00405 .

[15] E. Bernreuther, J. Horak, T. Plehn, and A. Butter, SciPost Phys. 5, 034 (2018).

[16] A. Titterton, U. Ellwanger, H. U. Flaecher, S. Moretti, and C. H. Shepherd-Themistocleous, J. High Energy Phys. 10 (2018) 064.

[17] S. Banerjee, B. Batell, and M. Spannowsky, Phys. Rev. D 95, 035009 (2017).

[18] S. Banerjee, F. Krauss, and M. Spannowsky, Phys. Rev. D 100, 073012 (2019).

[19] A. Adhikary, S. Banerjee, R. Kumar Barman, and B. Bhattacherjee, J. High Energy Phys. 09 (2019) 068.

[20] S. Banerjee, C. Englert, M. L. Mangano, M. Selvaggi, and M. Spannowsky, Eur. Phys. J. C 78, 322 (2018).

[21] A. M. Sirunyan et al. (CMS Collaboration), Phys. Rev. D 97, 032007 (2018).

[22] A. M. Sirunyan et al. (CMS Collaboration), Phys. Rev. Lett. 120, 241801 (2018).
[23] M. Aaboud et al. (ATLAS Collaboration), Phys. Rev. D 98, 092002 (2018).

[24] M. Blanke, S. Kast, J. M. Thompson, S. Westhof, and J. Zurita, J. High Energy Phys. 04 (2019) 160.

[25] J. S. Kim, O. Lebedev, and D. Schmeier, J. High Energy Phys. 11 (2015) 128.

[26] C. Gross, O. Lebedev, and Y. Mambrini, J. High Energy Phys. 08 (2015) 158.

[27] T. Hambye, J. High Energy Phys. 01 (2009) 028.

[28] O. Lebedev, H. M. Lee, and Y. Mambrini, Phys. Lett. B 707, 570 (2012).

[29] D. Buttazzo, L. Di Luzio, G. Landini, A. Strumia, and D. Teresi, J. High Energy Phys. 10 (2019) 067.

[30] D. Buttazzo, L. Di Luzio, P. Ghorbani, C. Gross, G. Landini, A. Strumia, D. Teresi, and J. W. Wang, J. High Energy Phys. 01 (2020) 130.

[31] A. Karam and K. Tamvakis, Phys. Rev. D 94, 055004 (2016).

[32] A. Poulin and S. Godfrey, Phys. Rev. D 99, 076008 (2019).

[33] G. Arcadi, C. Gross, O. Lebedev, S. Pokorski, and T. Toma, Phys. Lett. B 769, 129 (2017).

[34] G. Arcadi, C. Gross, O. Lebedev, Y. Mambrini, S. Pokorski, and T. Toma, J. High Energy Phys. 12 (2016) 081.

[35] K. Huitu, N. Koivunen, O. Lebedev, S. Mondal, and T. Toma, Phys. Rev. D 100, 015009 (2019).

[36] A. Falkowski, C. Gross, and O. Lebedev, J. High Energy Phys. 05 (2015) 057,

[37] T. Robens and T. Stefaniak, Eur. Phys. J. C 75, 104 (2015).

[38] A. Djouadi, O. Lebedev, Y. Mambrini, and J. Quevillon, Phys. Lett. B 709, 65 (2012).

[39] A. Djouadi, A. Falkowski, Y. Mambrini, and J. Quevillon, Eur. Phys. J. C 73, 2455 (2013).

[40] C. H. Chen and T. Nomura, Phys. Rev. D 93, 074019 (2016).

[41] A. Adhikary, S. Banerjee, R. K. Barman, B. Bhattacherjee, and S. Niyogi, J. High Energy Phys. 07 (2018) 116.

[42] A. Alves, T. Ghosh, and F. S. Queiroz, Phys. Rev. D 100, 036012 (2019).

[43] A. M. Sirunyan et al. (CMS Collaboration), Phys. Lett. B 788, 7 (2019).

[44] ATLAS Collaboration, CERN Report No. ATL-PHYSPUB-2016-026, 2016. 
[45] J. Alwall, M. Herquet, F. Maltoni, O. Mattelaer, and T. Stelzer, J. High Energy Phys. 06 (2011) 128.

[46] J. Alwall, R. Frederix, S. Frixione, V. Hirschi, F. Maltoni, O. Mattelaer, H.-S. Shao, T. Stelzer, P. Torrielli, and M. Zaro, J. High Energy Phys. 07 (2014) 079.

[47] R. D. Ball et al., Nucl. Phys. B867, 244 (2013).

[48] R. D. Ball et al. (NNPDF Collaboration), J. High Energy Phys. 04 (2015) 040.

[49] T. Sjostrand, S. Mrenna, and P. Z. Skands, J. High Energy Phys. 05 (2006) 026.

[50] T. Sjostrand, S. Ask, J. R. Christiansen, R. Corke, N. Desai, P. Ilten, S. Mrenna, S. Prestel, C. O. Rasmussen, and P. Z. Skands, Comput. Phys. Commun. 191, 159 (2015).

[51] S. Hoeche, F. Krauss, N. Lavesson, L. Lonnblad, M. Mangano, A. Schalicke, and S. Schumann, arXiv:hep-ph/0602031.

[52] M. L. Mangano, M. Moretti, F. Piccinini, and M. Treccani, J. High Energy Phys. 01 (2007) 013.

[53] J. de Favereau, C. Delaere, P. Demin, A. Giammanco, V. Lemaître, A. Mertens, and M. Selvaggi (DELPHES3 Collaboration), J. High Energy Phys. 02 (2014) 057.

[54] M. Selvaggi, J. Phys. Conf. Ser. 523, 012033 (2014).

[55] A. Mertens, J. Phys. Conf. Ser. 608, 012045 (2015).
[56] M. Cacciari, G. P. Salam, and G. Soyez, Eur. Phys. J. C 72, 1896 (2012).

[57] M. Cacciari, G. P. Salam, and G. Soyez, J. High Energy Phys. 04 (2008) 063.

[58] ATLAS Collaboration, CERN Report No. ATL-PHYSPUB-2015-022, 2015.

[59] T. Chen and C. Guestrin, in KDD '16: Proceedings of the 22nd ACM SIGKDD International Conference on Knowledge Discovery and Data Mining (2016), pp. 785-794, https://doi.org/10.1145/2939672.2939785.

[60] P. Baldi, P. Sadowski, and D. Whiteson, Nat. Commun. 5, 4308 (2014).

[61] K. Y. Oyulmaz, A. Senol, H. Denizli, and O. Cakir, Phys. Rev. D 99, 115023 (2019).

[62] B. Bhattacherjee, S. Mukherjee, and R. Sengupta, J. High Energy Phys. 11 (2019) 156.

[63] N. Bakhet, M. Y. Khlopov, and T. Hussein, arXiv:1507 .06547.

[64] R. D. Field, Y. Kanev, M. Tayebnejad, and P. A. Griffin, Phys. Rev. D 53, 2296 (1996).

[65] G. Aad et al. (ATLAS Collaboration), Phys. Lett. B 801, 135145 (2020). 